\title{
PERIODIC ORBITS AND THEIR STABILITY IN THE RÖSSLER PROTOTYPE-4 SYSTEM
}

\author{
Isaac A. García ${ }^{a}$, Jaume Llibre $^{b}$ And Susanna Maza ${ }^{a}$ \\ ${ }^{a}$ Departament de Matemàtica, Universitat de Lleida, Avda. Jaume II, 69, 25001 \\ Lleida, Catalonia, Spain, \\ garcia@matematica.udl.cat, smaza@matematica.udl.cat \\ ${ }^{b}$ Departament de Matemàtiques, Universitat Autònoma de Barcelona, 08193 \\ Bellaterra, Barcelona, Catalonia, Spain, \\ jllibre@mat.uab.cat. \\ Corresponding author. Tel. +34935811303 . Fax +34935812790 .
}

\section{Introduction AND STATEMENT OF THE RESUlts}

O.E. Rössler inspired by the geometry of 3-dimensional flows, introduced several systems in the 1970s as prototypes of the the simplest autonomous differential equations having chaos, the simplicity is in the sense of minimal dimension, minimal number of parameters and minimal nonlinearities. In MathSciNet appear at this moment more than 165 articles about the Rössler's systems, some recent ones are $[1,2,4,7]$.

Here we consider the Rössler prototype-4 system

$$
\dot{x}=-y-z, \quad \dot{y}=x, \quad \dot{z}=\alpha y(1-y)-\beta z,
$$

introduced in [3]. See also the book [6]. This differential system exhibits chaotic motion for the parameter values around $\alpha=\beta=1 / 2$, having an strange attractor. In [6] it is numerically showed that in the region of the parameter space giving by small positive values of $\alpha$ and $\beta$ there are periodic orbits of (1), see Figure 3.8 in page 69 of [6]. As far as we know, this is the first time that an analytic analysis on the existence and stability of such periodic orbits of (1) is performed.

Theorem 1. Consider the Rössler prototype-4 system (1) satisfying $(\alpha+2 \beta) \alpha>0$. Consider the new parameters $(a, b)$ defined as follows $\alpha=\varepsilon a$ and $\beta=-\varepsilon\left(b+\frac{a}{1+\varepsilon^{2} b^{2}}\right)$. Then for $\varepsilon>0$ sufficiently small there exists one periodic solution $\gamma_{\varepsilon}$ of (1) such that $\gamma_{\varepsilon} \rightarrow\left\{z=-\frac{1}{2}\right\} \cap\left\{x^{2}+(y+z)^{2}=-\frac{a+2 b}{2 a}\right\}$ as $\varepsilon \rightarrow 0$. Moreover, $\gamma_{\varepsilon}$ is asymptotically stable when $a+b<0$, and unstable when $a+b>0$.

Theorem 1 is proved in section 2 .

Theorem 2. Consider the Rössler prototype-4 system (1) satisfying $\alpha>2 \beta^{3}$. Consider the new parameters $(a, \varepsilon)$ defined by $\alpha=\varepsilon\left(2 a-1+a^{2} \varepsilon^{2}\right)$ and $\beta=\varepsilon(1-a)$. Then for $\varepsilon>0$ sufficiently small and $a>1 / 2$ there exists one periodic solution $\gamma_{\varepsilon}$ of (1) such that $\gamma_{\varepsilon} \rightarrow\left\{z=-\frac{1}{2}\right\} \cap\left\{x^{2}+(y+z)^{2}=\frac{1}{2(2 a-1)}\right\}$ as $\varepsilon \rightarrow 0$. Moreover, $\gamma_{\varepsilon}$ is asymptotically stable when $\frac{1}{2}<a<1$, and unstable when $a>1$.

Theorem 2 is proved in section 3 .

The main tool for proving our theorems is the averaging theory of first order described in the appendix. 


\section{Proof of Theorem 1}

Since $\alpha=\varepsilon a$ and $\beta=-\varepsilon\left(b+\frac{a}{1+\varepsilon^{2} b^{2}}\right) \approx-\varepsilon(a+b)$, if $(\alpha+2 \beta) \alpha>0$ and $\varepsilon$ is sufficiently small, then

$$
(a+2 b) a<0
$$

because $(\alpha+2 \beta) \alpha \approx-\varepsilon^{2}(a+2 b) a$.

Now system (1) writes

$$
\dot{x}=-y-z, \quad \dot{y}=x, \quad \dot{z}=\varepsilon a y(1-y)+\varepsilon\left(b+\frac{a}{1+\varepsilon^{2} b^{2}}\right) z .
$$

The characteristic polynomial $p(\lambda)$ of the linearization of system (3) at the equilibrium point located at the origin is

$$
p(\lambda)=\frac{(\lambda-\varepsilon b)\left[-1+\varepsilon^{2} b(a-b)+a \varepsilon \lambda-\left(1+\varepsilon^{2} b^{2}\right) \lambda^{2}\right]}{1+\varepsilon^{2} b^{2}} .
$$

Therefore, when $\varepsilon \rightarrow 0$ the eigenvalues associated at the origin are 0 and $\pm i$ with $i^{2}=-1$. Doing the linear change of variables

$$
\left(\begin{array}{l}
X \\
Y \\
Z
\end{array}\right)=\left(\begin{array}{ccc}
0 & -1 & -1 \\
1 & 0 & 0 \\
0 & 0 & 1
\end{array}\right)\left(\begin{array}{l}
x \\
y \\
z
\end{array}\right),
$$

the linear part at the origin of system (3) with $\varepsilon=0$ becomes in its real Jordan normal form

$$
\left(\begin{array}{ccc}
0 & -1 & 0 \\
1 & 0 & 0 \\
0 & 0 & 0
\end{array}\right)
$$

and system (3) writes

(4) $\dot{X}=-Y+\varepsilon f(X, Y, Z)+O\left(\varepsilon^{2}\right), \quad \dot{Y}=X, \quad \dot{Z}=-\varepsilon f(X, Y, Z)+O\left(\varepsilon^{2}\right)$, with $f(X, Y, Z)=-b Z+a\left(X+X^{2}+2 X Z+Z^{2}\right)$.

Now we perform the change to cylindrical coordinates $(X, Y, Z) \mapsto(\theta, r, z)$ with $x=r \cos \theta, y=r \sin \theta$ and $z=Z$. Then system (4) becomes

$$
\begin{aligned}
\dot{r} & =\varepsilon g_{1}(\theta, r, z)+O\left(\varepsilon^{2}\right), \\
\dot{\theta} & =1+\varepsilon g_{2}(\theta, r, z)+O\left(\varepsilon^{2}\right), \\
\dot{z} & =\varepsilon g_{3}(x, y, z)+O\left(\varepsilon^{2}\right),
\end{aligned}
$$

with

$$
\begin{aligned}
& g_{1}(\theta, r, z)=-\cos \theta g_{3}(\theta, r, z), \\
& g_{2}(\theta, r, z)=\frac{\sin \theta}{r} g_{3}(\theta, r, z), \\
& g_{3}(\theta, r, z)=b z-a\left(z^{2}+r \cos \theta(1+2 z+r \cos \theta)\right) .
\end{aligned}
$$

Notice that this system is only well defined for $r>0$. Moreover, in this region, since for sufficiently small $\varepsilon$ we have $\dot{\theta}>0$ in a big ball centered at the origin, we can rewrite the differential system (5) in this big ball into the form

$$
\frac{d r}{d \theta}=\varepsilon g_{1}(\theta, r, z)+O\left(\varepsilon^{2}\right), \frac{d z}{d \theta}=\varepsilon g_{3}(\theta, r, z)+O\left(\varepsilon^{2}\right),
$$


by taking $\theta$ as the new independent variable. System (6) is $2 \pi$-periodic in the variable $\theta$ and is in the standard form for applying the averaging theory, see Theorem 3 of the appendix. The average of $g_{1}$ and $g_{3}$ with respect to $\theta$ are

$$
\begin{aligned}
& \overline{g_{1}}(r, z)=\frac{1}{2 \pi} \int_{0}^{2 \pi} g_{1}(\theta, r, z) d \theta=\frac{1}{2} \operatorname{ar}(1+2 z), \\
& \overline{g_{3}}(r, z)=\frac{1}{2 \pi} \int_{0}^{2 \pi} g_{3}(\theta, r, z) d \theta=\frac{1}{2}\left[-a\left(r^{2}+2 z^{2}\right)+2 b z\right] .
\end{aligned}
$$

The only zero $\left(r^{*}, z^{*}\right)$ with $r^{*}>0$ of the map $(r, z) \mapsto \mathcal{F}(r, z)=\left(\overline{g_{1}}(r, z), \overline{g_{3}}(r, z)\right)$ is

$$
\left(r^{*}, z^{*}\right)=\left(\sqrt{-\frac{a+2 b}{2 a}},-\frac{1}{2}\right) .
$$

Notice that $r^{*} \in \mathbb{R}$ because of (2). In addition the Jacobian matrix of $\mathcal{F}$ at the point $\left(r^{*}, z^{*}\right)$ is

$$
D \mathcal{F}\left(r^{*}, z^{*}\right)=\left(\begin{array}{cc}
0 & \sqrt{-\frac{(a+2 b) a}{2}} \\
-\sqrt{-\frac{(a+2 b) a}{2}} & a+b
\end{array}\right),
$$

and its determinant is

$$
\operatorname{det}\left(D \mathcal{F}\left(r^{*}, z^{*}\right)\right)=-\frac{1}{2} a(a+2 b)>0,
$$

again from $(2)$. Therefore $\left(r^{*}, z^{*}\right)$ is a simple zero of $\mathcal{F}$. Hence, the averaging theory of Theorem 3 predicts the existence of a $2 \pi$-periodic orbit $\gamma_{\varepsilon}=\{(r(\theta, \varepsilon), z(\theta, \varepsilon))$ : $0 \leq \theta \leq 2 \pi\}$ of system (6) such that $(r(0, \varepsilon), z(0, \varepsilon)) \rightarrow\left(r^{*}, z^{*}\right)$ as $\varepsilon \rightarrow 0$.

In addition, denoting by $\lambda_{1}$ and $\lambda_{2}$ the eigenvalues of $D \mathcal{F}\left(r^{*}, z^{*}\right)$, we obtain that

$$
\lambda_{1} \lambda_{2}=\operatorname{det}\left(D \mathcal{F}\left(r^{*}, z^{*}\right)\right)>0, \quad \lambda_{1}+\lambda_{2}=a+b .
$$

Therefore, from Theorem 3 the periodic orbit $\gamma_{\varepsilon}$ is either stable if $a+b<0$, or unstable if $a+b>0$. Hence the conclusion of the theorem about the stability of $\gamma_{\varepsilon}$ is proved.

Now we go back along the performed changes of variables and time rescaling which keep the stability of the periodic orbit $\gamma_{\varepsilon}$. Thus we have that system (4) has a periodic orbit $\gamma_{\varepsilon}$ such that $\gamma_{\varepsilon} \rightarrow C$ as $\varepsilon \rightarrow 0$, where $C$ is the circle given by $C=\left\{Z=z^{*}\right\} \cap\left\{X^{2}+Y^{2}=r^{* 2}\right\}$, the intersection of a plane and a cylinder. Finally, system (3) and therefore the Rössler prototype-4 system (1) has a periodic orbit $\gamma_{\varepsilon}$ such that $\gamma_{\varepsilon} \rightarrow\left\{z=z^{*}\right\} \cap\left\{x^{2}+(y+z)^{2}=r^{* 2}\right\}$ as $\varepsilon \rightarrow 0$.

\section{Proof of Theorem 2}

Since $\alpha=\varepsilon\left(2 a-1+a^{2} \varepsilon^{2}\right)$ and $\beta=\varepsilon(1-a)$ system (1) writes

$$
\dot{x}=-y-z, \quad \dot{y}=x, \quad \dot{z}=\varepsilon\left[\left(2 a-1+a^{2} \varepsilon^{2}\right) y(1-y)-(1-a) z\right] .
$$

System (7) has besides the origin the equilibrium point

$$
\left(x_{e}, y_{e}, z_{e}\right)=\left(0, \frac{a\left(1+a \varepsilon^{2}\right)}{a\left(2+a \varepsilon^{2}\right)-1}, \frac{a-1}{a\left(2+a \varepsilon^{2}\right)-1}-1\right),
$$


which always exists for sufficiently small values of $|\varepsilon|$ if we take $a \neq 1 / 2$. The characteristic polynomial $p(\lambda)$ of the linearization of system (7) at the equilibrium point $\left(x_{e}, y_{e}, z_{e}\right)$ is given by

$$
p(\lambda)=(a \varepsilon-\lambda)\left(\lambda^{2}+\varepsilon \lambda+a \varepsilon^{2}+1\right) .
$$

Therefore, when $\varepsilon \rightarrow 0$ the eigenvalues associated to $\left(x_{e}, y_{e}, z_{e}\right)$ are 0 and $\pm i$ with $i^{2}=-1$. After translating the equilibrium point $\left(x_{e}, y_{e}, z_{e}\right)$ to the origin through the change of variables $(x, y, z) \mapsto\left(x-x_{e}, y-y_{e}, z-z_{e}\right)$ system (7) becomes

(8) $\dot{x}=-y-z, \quad \dot{y}=x, \quad \dot{z}=-\varepsilon\left[\left(1+a^{2} \varepsilon^{2}\right) y+(1-a) z+\left(2 a-1+a^{2} \varepsilon^{2}\right) y^{2}\right]$.

Performing the linear change of variables

$$
\left(\begin{array}{l}
X \\
Y \\
Z
\end{array}\right)=\left(\begin{array}{lll}
1 & 0 & 0 \\
0 & 1 & 1 \\
0 & 0 & 1
\end{array}\right)\left(\begin{array}{l}
x \\
y \\
z
\end{array}\right),
$$

we transform system (8) into a system having its linear part at the origin in its real Jordan canonical form when $\varepsilon \rightarrow 0$. More precisely, in these new linear coordinates system (8) becomes

(9) $\quad \dot{X}=-Y, \quad \dot{Y}=X+\varepsilon f(X, Y, Z)+O\left(\varepsilon^{2}\right), \quad \dot{Z}=\varepsilon f(X, Y, Z)+O\left(\varepsilon^{2}\right)$,

with

$$
f(X, Y, Z)=(1-2 a) Y^{2}+Z(a+Z-2 a Z)+Y[2 Z(2 a-1)-1] .
$$

Now we perform the change to cylindrical coordinates $(X, Y, Z) \mapsto(\theta, r, z)$ with $x=r \cos \theta, y=r \sin \theta$ and $z=Z$. Then system (9) writes

$$
\begin{aligned}
\dot{r} & =\varepsilon g_{1}(\theta, r, z)+O\left(\varepsilon^{2}\right), \\
\dot{\theta} & =1+\varepsilon g_{2}(\theta, r, z)+O\left(\varepsilon^{2}\right), \\
\dot{z} & =\varepsilon g_{3}(x, y, z)+O\left(\varepsilon^{2}\right),
\end{aligned}
$$

with

$$
\begin{aligned}
& g_{1}(\theta, r, z)=\sin \theta g_{3}(\theta, r, z), \\
& g_{2}(\theta, r, z)=\frac{\cos \theta}{r} g_{3}(\theta, r, z), \\
& g_{3}(\theta, r, z)=z(a+z-2 a z)+r \sin \theta[2(2 a-1) z+r(1-2 a) \sin \theta-1] .
\end{aligned}
$$

This system is only well defined for $r>0$. Moreover, in this region, since for sufficiently small $\varepsilon$ we have $\dot{\theta}>0$ in a big ball centered at the origin, we can rewrite the differential system(10) in this big ball into the form

$$
\frac{d r}{d \theta}=\varepsilon g_{1}(\theta, r, z)+O\left(\varepsilon^{2}\right), \quad \frac{d z}{d \theta}=\varepsilon g_{3}(\theta, r, z)+O\left(\varepsilon^{2}\right),
$$

taking $\theta$ as the new independent variable. System (11) is $2 \pi$-periodic in the variable $\theta$ and is in the standard form for applying the averaging theory, see Theorem 3. The average of $g_{1}$ and $g_{2}$ with respect to $\theta$ are

$$
\begin{aligned}
& \overline{g_{1}}(r, z)=\frac{1}{2 \pi} \int_{0}^{2 \pi} g_{1}(\theta, r, z), d \theta=\frac{1}{2} r(2 z(2 a-1)-1), \\
& \overline{g_{3}}(r, z)=\frac{1}{2 \pi} \int_{0}^{2 \pi} g_{3}(\theta, r, z) d \theta=\frac{1}{2}\left[r^{2}(1-2 a)+2 z(a+z-2 a z)\right] .
\end{aligned}
$$


The only zero $\left(r^{*}, z^{*}\right)$ of the map $(r, z) \mapsto \mathcal{F}(r, z)=\left(\overline{g_{1}}(r, z), \overline{g_{3}}(r, z)\right)$ with $r^{*}>0$ is

$$
\left(r^{*}, z^{*}\right)=\left(\frac{1}{\sqrt{2(2 a-1)}}, \frac{1}{2(2 a-1)}\right),
$$

which only exists under the extra condition

$$
a>\frac{1}{2} .
$$

This condition implies $\alpha>\varepsilon^{3} / 4$ and $\beta<\varepsilon / 2$ from where it follows the initial parameter restriction $\alpha>2 \beta^{3}$. In addition the Jacobian matrix of $\mathcal{F}$ at the point $\left(r^{*}, z^{*}\right)$ is

$$
D \mathcal{F}\left(r^{*}, z^{*}\right)=\left(\begin{array}{cc}
0 & \sqrt{a-\frac{1}{2}} \\
-\sqrt{a-\frac{1}{2}} & a-1
\end{array}\right),
$$

and its determinant is

$$
\operatorname{det}\left(D \mathcal{F}\left(r^{*}, z^{*}\right)\right)=a-\frac{1}{2}>0 .
$$

Therefore $\left(r^{*}, z^{*}\right)$ is a simple zero of $\mathcal{F}$. Hence, the averaging theory of Theorem 3 predicts the existence of a $2 \pi$-periodic orbit $\gamma_{\varepsilon}=\{(r(\theta, \varepsilon), z(\theta, \varepsilon)): 0 \leq \theta \leq 2 \pi\}$ of system (11) such that $(r(0, \varepsilon), z(0, \varepsilon)) \rightarrow\left(r^{*}, z^{*}\right)$ as $\varepsilon \rightarrow 0$.

Since the trace of the matrix (13) is $a-1$ and its determinat is positive when $a>1 / 2$, it follows from Theorem 3 that the periodic orbit $\gamma_{\varepsilon}$ is either asymptotically stable if $1 / 2<a<1$ and unstable if $a>1$. Hence the conclusion of the theorem about the stability of $\gamma_{\varepsilon}$ is proved.

Now we go back along the performed changes of variables and time rescaling which keep the stability of the periodic orbit $\gamma_{\varepsilon}$. Thus we have that system (9) has one periodic orbit $\gamma_{\varepsilon}$ such that $\gamma_{\varepsilon} \rightarrow C$ as $\varepsilon \rightarrow 0$, where $C$ is the circle given by $C=\left\{Z=z^{*}\right\} \cap\left\{X^{2}+Y^{2}=r^{* 2}\right\}$, the intersection of a plane and a cylinder. Finally, system (7) and therefore the Rössler prototype- 4 system (1) has one periodic orbit $\gamma_{\varepsilon}$ such that $\gamma_{\varepsilon} \rightarrow\{z=-1 / 2\} \cap\left\{x^{2}+(y+z)^{2}=1 /(2(2 a-1))\right\}$ as $\varepsilon \rightarrow 0$. This completes the proof of Theorem 2 .

\section{The APPEndix: Averaging theOry OF FIRST ORDER}

Now we shall present the basic results from averaging theory that we need for proving the results of this paper. The next theorem provides a first order approximation for the periodic solutions of a periodic differential system, for a proof see Theorems 11.5 and 11.6 of Verhulst [8]. For more details on the averaging theory see [5].

Consider the differential equation in the standard form

$$
\dot{\mathbf{x}}=\varepsilon F_{1}(t, \mathbf{x})+\varepsilon^{2} F_{2}(t, \mathbf{x}, \varepsilon), \quad \mathbf{x}(0)=\mathbf{x}_{0}
$$

with $\mathbf{x} \in D$, where $D$ is an open subset of $\mathbb{R}^{n}, t \geq 0$. Moreover we assume that both $F_{1}(t, \mathbf{x})$ and $F_{2}(t, \mathbf{x}, \varepsilon)$ are $T$-periodic in $t$. We also consider in $D$ the averaged differential equation

$$
\dot{\mathbf{y}}=\varepsilon f_{1}(\mathbf{y}), \quad \mathbf{y}(0)=\mathbf{x}_{0},
$$


where

$$
f_{1}(\mathbf{y})=\frac{1}{T} \int_{0}^{T} F_{1}(t, \mathbf{y}) d t .
$$

Under certain conditions, equilibrium solutions of the averaged equation turn out to correspond with $T$-periodic solutions of equation (14).

Theorem 3. Consider the two initial value problems (14) and (15). Suppose:

(i) $F_{1}$, its Jacobian $\partial F_{1} / \partial \mathbf{x}$, its Hessian $\partial^{2} F_{1} / \partial \mathbf{x}^{2}, F_{2}$ and its Jacobian $\partial F_{2} / \partial \mathbf{x}$ are defined, continuous and bounded by a constant independent of $\varepsilon$ in $[0, \infty) \times D$ and $\varepsilon \in\left(0, \varepsilon_{0}\right]$.

(ii) $F_{1}$ and $F_{2}$ are $T$-periodic in $t$ ( $T$ independent of $\varepsilon$ ).

Then the following statements hold.

(a) If $p$ is an equilibrium point of the averaged equation (15) and

$$
\left.\operatorname{det}\left(\frac{\partial f_{1}}{\partial \mathbf{y}}\right)\right|_{\mathbf{y}=p} \neq 0,
$$

then there exists a T-periodic solution $\varphi(t, \varepsilon)$ of equation (14) such that $\varphi(0, \varepsilon) \rightarrow p$ as $\varepsilon \rightarrow 0$.

(b) If the eigenvalues of the equilibrium point $p$ all have negative real part, the corresponding periodic orbit $\varphi(t, \varepsilon)$ is asymptotically stable for $\varepsilon$ sufficiently small. If one of the eigenvalues has positive real part, then $\varphi(t, \varepsilon)$ is unstable.

\section{ACKNOWLEDGEMENTS}

The first and third authors are partially supported by a MICINN/FEDER grant number MTM2008-00694 and by an AGAUR grant number 2009SGR 381. The second author is partially supported by a MICINN/FEDER grant number MTM200803437, by an AGAUR grant number 2009SGR 410 and by ICREA Academia.

\section{REFERENCES}

[1] E. Ahmed, A.M.A. El-Sayed, H.A.A. El-Saka, Phys. Lett. A 358 (2006) 1.

[2] Q. Li, Phys. Lett. A 372 (2008) 2989.

[3] O.E. Rössler, Ann. New York Acad. Sci. 316 (1979) 376.

[4] L. Runzi, Phys. Lett. A 372 (2008) 3667.

[5] J.A. Sanders, F. Verhulst, J. Murdock, Averaging methods in nonlinear dynamical systems, Second edition, Applied Mathematical Sciences, 59, Springer, New York, 2007.

[6] J.C. Sprott, Elegant chaos. Algebraically simple chaotic flows, World Scientific Publishing, 2010 .

[7] A. Szczepaniak, W.M. Macek, Phys. Lett. A 372 (2008) 2423.

[8] F. Verhulst, Nonlinear Differential Equations and Dynamical Systems, Universitext, Springer, 1991. 